Service social

\title{
Vivre une expérience de soins à domicile, par Mario Paquet, Québec, Les Presses de l'Université Laval, 2003, 230 p.
}

\section{Majorie Poulin}

Volume 52, numéro 1, 2006

URI : https://id.erudit.org/iderudit/015959ar

DOI : https://doi.org/10.7202/015959ar

Aller au sommaire du numéro

Éditeur(s)

École de service social de l'Université Laval

ISSN

1708-1734 (numérique)

Découvrir la revue

Citer ce compte rendu

Poulin, M. (2006). Compte rendu de [Vivre une expérience de soins à domicile, par Mario Paquet, Québec, Les Presses de l'Université Laval, 2003, 230 p.]

Service social, 52(1), 9-11. https://doi.org/10.7202/015959ar d'utilisation que vous pouvez consulter en ligne.

https://apropos.erudit.org/fr/usagers/politique-dutilisation/ 


\title{
RECENSION
}

\author{
Vivre une expérience de soins à domicile \\ par Mario Paquet \\ Québec, Les Presses de l'Université Laval, 2003, 230 p.
}

\begin{abstract}
Majorie Poulin
Bachelière en psychologie

Titulaire d'un certificat en gérontologie

Étudiante à la maîtrise en service social

Université Laval, Québec
\end{abstract}

L'auteur de cet ouvrage est sociologue. Depuis 1987, il est agent de recherche à la Direction de la santé publique et d'évaluation de la Régie régionale de la santé et des services sociaux de Lanaudière, au Québec. Il est également chercheur invité à I'Institut national de la recherche scientifique (INRS-Urbanisation, Culture et Société) et chercheur associé au centre de recherche de l'Institut universitaire de gériatrie de Montréal. Mario Paquet tente de mieux faire comprendre au lecteur la logique familiale qui sous-tend les soins auprès d'un proche âgé. II se penche ici sur la réalité quotidienne de l'aidant familial qu'est le conjoint ou la conjointe. II insiste sur l'importance de placer ce citoyen « au centre » du système de santé en lui donnant droit de parole.

Dans cette perspective, il a divisé son ouvrage de la façon suivante :

Le premier chapitre explore tout d'abord le concept d'aidant naturel. L'auteur y mentionne que l'utilisation de ce terme peut devenir un irritant pour l'aidant au sens où ce n'est pas dans la « nature des choses » d'accomplir quotidiennement les diverses responsabilités inhérentes aux soins requis par l'état de santé d'un proche âgé. Devant la lourdeur et la complexité des diverses tâches à réaliser en fonction de ce rôle, l'aidant familial se donne la liberté d'ajouter le suffixe « sur » au concept. Nous nous trouvons ainsi dorénavant en présence d'un(e) « aidant(e) surnaturel(le) », terme qui apparaît comme étant plus conforme à la réalité. Le premier chapitre s'intéresse également à la perception du système de santé par les aidants. L'auteur procède à une analyse critique de celui-ci en établissant les retombées négatives du virage ambulatoire qui se greffent aux contraintes vécues par les aidants. Nous retrouvons finalement la question du partenariat en maintien à domicile. Alors que le partenariat en maintien à domicile suppose des actions concertées pour arriver à une complémentarité dans la planification et l'organisation des services, la perception qu'en ont les aidants est plutôt dirigée vers l'humanisation des soins. L'auteur aborde les valeurs qui devraient s'inscrire dans un projet de partenariat que sont le respect, l'égalité de 
pouvoir et l'équité dans les efforts déployés. II en arrive à démontrer que celles-ci sont loin de correspondre à la relation qui existe entre l'État et les fournisseurs de soins informels.

Le deuxième chapitre aborde l'état des connaissances sur le soin familial aux personnes âgées dépendantes. Plus précisément, l'auteur y signale une tendance des travaux scientifiques dans ce champ à mettre l'accent plus sur la mesure que sur la compréhension. II critique en outre le fait que de nombreuses recherches étudient la réalité des familles sous l'angle des problèmes, montrant l'importance à accorder à l'expérience humaine vécue à l'intérieur du rôle d'aidant qui va au-delà du « fardeau » qui y est souvent associé. II s'attarde également à la complexité sous-jacente à la logique familiale, insistant sur la nécessité pour le chercheur de s'intégrer dans le quotidien des personnes qui vivent une expérience de soins pour avoir une meilleure compréhension de leur réalité et en saisir toute la complexité. Des avenues permettant de mieux comprendre la logique de soins auprès d'un proche aidant sont suggérées dans ce chapitre, où sont posées les questions du «pourquoi » et du «comment » d'une expérience de soins.

Les troisième et quatrième chapitres sont consacrés à la présentation, puis à la comparaison de deux expériences de soins par un proche. En cherchant à reconnaître les similitudes et les différences entre ces expériences, l'auteur fait l'éloge de l'amour, du courage et de la détermination qui émergent au-delà de la situation vécue. II s'intéresse à la signification que peut prendre l'expérience de soins dans la compréhension de l'engagement de l'aidant: les liens d'affection et l'expression de l'amour sont selon lui le « moteur d'action » dans l'aide. II est aussi question dans ce chapitre des apprentissages vécus de part et d'autre que sont les compromis ou encore les grands et les petits deuils auxquels les aidants et leur famille doivent faire face: éloignement de l'entourage, manque de soutien de la part de la famille et isolement. Enfin, l'auteur met en évidence l'importance du savoir-être dans le recours aux services formels, cette «culture du cœur » évoquant la dimension relationnelle dans les soins.

Dans le dernier chapitre, Paquet examine la « réticence », cette attitude de fermeture que l'on retrouve souvent chez les personnes-soutien face à l'utilisation des services. II parle ainsi de « la règle du dernier recours », qui fait en sorte que plusieurs personnessoutien ne font appel aux services formels qu'en cas de force majeure. Différents facteurs descriptifs et explicatifs sont ici abordés: l'écart d'utilisation entre services médicaux et services sociaux (à l'avantage des premiers); les différences d'utilisation en fonction de l'âge, du sexe et de la proximité relationnelle; la méconnaissance des services, l'organisation de ceux-ci et la logique familiale comme facteurs déterminants dans la recherche/non-recherche d'aide. Enfin, l'auteur souligne à maintes reprises le manque de confiance des personnes-soutien vis-à-vis des étrangers dans l'explication de la réticence, de même que le besoin de retrouver un lien de « proximité à distance » avec les professionnels travaillant dans le système. 
Cet ouvrage explore les multiples facettes propres à la réalité que représentent les soins familiaux aux personnes âgées à partir d'entretiens, de récits et d'analyses tout aussi fascinants les uns que les autres. L'auteur tente de mieux faire comprendre la logique familiale qui sous-tend les soins auprès d'un proche âgé en parlant d'acteurs qui se « démènent pour rester autonomes » ou pour faire en sorte que leurs proches le demeurent. Son objectif rejoint la recommandation du Conseil de la santé et du bienêtre qui est de «sensibiliser les intervenantes et les intervenants à la logique d'aide des familles, différente du réseau de services, et d'ajuster les pratiques pour une adaptation des services à la dynamique familiale qui respecte les besoins et les valeurs des personnes aidantes et la solidarité familiale » (Fr. Lacroix, 2001, p. 30). II nous apparaît que ce livre peut apporter des pistes de solution pour que le maintien à domicile soit davantage orienté vers la qualité des liens entre humains, élément essentiel à l'utilisation des services. 